\title{
Significance of H3K27me3 loss in the diagnosis of malignant peripheral nerve sheath tumors
}

\author{
Melike Pekmezci ${ }^{1}$, Areli K Cuevas-Ocampo ${ }^{1}$, Arie Perry ${ }^{1,2}$ and Andrew E Horvai ${ }^{1}$ \\ ${ }^{1}$ Department of Pathology, University of California San Francisco, San Francisco, CA, USA and ${ }^{2}$ Department \\ of Neurological Surgery, University of California San Francisco, San Francisco, CA, USA
}

\begin{abstract}
The diagnosis of malignant peripheral nerve sheath tumors can be challenging and other spindle cell sarcomas commonly enter in the differential diagnosis. Loss of trimethylation at lysine 27 of histone-H3 (H3K27me3) by immunohistochemistry was recently described in malignant peripheral nerve sheath tumors. However, its specificity remains controversial. We therefore studied 82 synovial sarcomas, 39 malignant peripheral nerve sheath tumors, and 10 fibrosarcomatous dermatofibrosarcoma protuberans for H3K27me3 loss by immunohistochemistry. The diagnoses were based on morphology, immunophenotype, and genetics based on WHO classification. H3K27me3 immunohistochemistry was scored by two pathologists based on fraction of cells with nuclear staining (score 0 to 3+). Loss of $\mathrm{H} 3 \mathrm{~K} 27 \mathrm{me} 3$ (score 0 ) was seen in $44 \%$ of malignant peripheral nerve sheath tumors and $9 \%$ of synovial sarcomas yielding positive and negative predictive values of $71 \%$ and $77 \%$, respectively. Loss of $\mathrm{H} 3 \mathrm{~K} 27 \mathrm{me} 3$ was seen in $10 \%$ of fibrosarcomatous dermatofibrosarcoma protuberans, yielding positive and negative predictive values of 94 and $29 \%$ in the differential diagnosis of malignant peripheral nerve sheath tumor versus fibrosarcomatous dermatofibrosarcoma protuberans. Partial loss (score 1-2) was common in all three tumor types. Among malignant peripheral nerve sheath tumors, there was no significant association between $\mathrm{H} 3 \mathrm{~K} 27 \mathrm{me} 3$ loss and gender, tumor site, or size, and progression-free or overall survival. Patients with tumors with $\mathrm{H} 3 \mathrm{~K} 27 \mathrm{me} 3$ loss were younger than those with tumors with retained H3K27me3 expression $(P=0.011)$. H3K27me3 expression was lost in 50 and $31 \%$ of sporadic and Neurofibromatosis-associated malignant peripheral nerve sheath tumors, respectively $(P=0.25)$. Complete H3K27me3 loss is a moderately sensitive and relatively specific marker for the diagnosis of malignant peripheral nerve sheath tumor when the differential diagnosis includes synovial sarcoma and fibrosarcomatous dermatofibrosarcoma protuberans. Partial loss has limited diagnostic utility. H3K27me3 status does not show significant association with clinical outcome in malignant peripheral nerve sheath tumors.
\end{abstract}

Modern Pathology (2017) 30, 1710-1719; doi:10.1038/modpathol.2017.97; published online 4 August 2017

Malignant peripheral nerve sheath tumors are rare sarcomas, predominantly seen in deep soft tissues of adults, either in the setting of neurofibromatosis type-1 or sporadically. ${ }^{1}$ The majority of malignant peripheral nerve sheath tumors are conventional spindle cell neoplasms demonstrating characteristic intersecting fascicles, high mitotic rate and geographic necrosis. ${ }^{2}$ However; the histologic features are not diagnostically specific, particularly in the absence of intraneural growth, clinical history of

Correspondence: Dr M Pekmezci, MD, University of California San Francisco, Department of Pathology, Division of Neuropathology, 505 Parnassus Ave, Room M551, Box: 0102, San Francisco, CA 94143, USA.

E-mail: Melike.Pekmezci@ucsf.edu

Received 29 March 2017; revised 13 June 2017; accepted 18 June 2017; published online 4 August 2017
Neurofibromatosis, or a precursor neurofibroma. Frequently, other spindle cell sarcomas including synovial sarcoma and fibrosarcomatous dermatofibrosarcoma protuberans enter in the differential diagnosis, especially in the setting of sporadic tumors. While malignant peripheral nerve sheath tumors may show positive staining with neural crest cell markers such as S100 and SOX10, these markers are not very sensitive and are not entirely specific, leading to further diagnostic challenges. ${ }^{3,4}$

Recent studies showed inactivation of polycomb repressive complex 2 (PRC2) in a large subset of malignant peripheral nerve sheath tumors, due to loss-of-function mutations in PRC2 subunits EED or SUZ12. ${ }^{5,6}$ These co-occur with somatic mutations of CDKN2A and NF1, and are associated with a distinct DNA methylation profile. ${ }^{6,7}$ In addition to its DNA-wide effects on methylation, PRC2 inactivation 
specifically leads to loss of trimethylation at lysine 27 of histone-H3 (H3K27me3). The loss of methylation can be demonstrated by highly specific immunohistochemistry. ${ }^{6,8}$ Initial studies have been encouraging in the utility of H3K27me3 loss in the diagnosis of malignant peripheral nerve sheath tumor compared to morphologic mimics. ${ }^{6-11}$ However, the results for synovial sarcoma and fibrosarcomatous dermatofibrosarcoma protuberans have varied among studies with some reporting near $100 \%$ specificity and others reporting relatively poor specificity when compared to malignant peripheral nerve sheath tumor. ${ }^{8-11}$ Given that monophasic synovial sarcoma and fibrosarcomatous dermatofibrosarcoma protuberans are usually monomorphic, hypercellular spindle cell sarcomas with fascicular growth patterns, they represent some of the best morphologic mimics of malignant peripheral nerve sheath tumor. Consequently, H3K27me3 specificity is vital to the diagnostic utility of the stain when attempting to exclude synovial sarcoma and fibrosarcomatous dermatofibrosarcoma protuberans.

The main aim of the present study is to evaluate H3K27me3 staining pattern in malignant peripheral nerve sheath tumor, synovial sarcoma and fibrosarcomatous dermatofibrosarcoma protuberans diagnosed in our institution and assess its utility in this differential diagnosis.

\section{Materials and methods}

\section{Selection of Cases}

We searched the Department of Pathology archives at University of California San Francisco for primary malignant peripheral nerve sheath tumors and synovial sarcomas diagnosed between January 1991 and December 2012. After confirmation of the diagnoses, 83 synovial sarcoma, 40 malignant peripheral nerve sheath tumor, and 10 fibrosarcomatous dermatofibrosarcoma protuberans cases with sufficient pathology material were considered for the study. One synovial sarcoma and one malignant peripheral nerve sheath tumor were subsequently excluded due to failed H3K27me3 immunohistochemical stain in the absence of internal positive control. Sixty-two synovial sarcomas $(76 \%)$ were monophasic, two $(2 \%)$ were poorly differentiated, and 18 (22\%) were biphasic. We did not have any epithelioid malignant peripheral nerve sheath tumor or malignant peripheral nerve sheath tumor with glands in the current study. None of the sporadic malignant peripheral nerve sheath patients have history of radiation to suggest a radiation-induced sarcoma. Two of the Neurofibromatosis type- 1 patients had prior radiation. Subsets of the synovial sarcomas and malignant peripheral nerve sheath tumors have been previously published. ${ }^{3,12}$ Clinical information was collected from the electronic medical records. Outcome data including recurrencefree survival and overall survival were available only for malignant peripheral nerve sheath tumor cases.
The diagnoses were based on morphology, immunohistochemical profile, and genetic findings. All malignant peripheral nerve sheath tumor cases were independently reviewed by three study authors (MP, $\mathrm{AP}$, and $\mathrm{AEH}$ ) and only those with consensus diagnosis using the revised 4th edition of World Health Organization (WHO) Classification of the Tumors of the Central Nervous System were included in the study. ${ }^{13}$ Tumors arising from a nearby or previous neurofibroma, tumors involving a major nerve either by imaging, gross evaluation or microscopic assessment, tumors demonstrating Schwannian differentiation by S100 and/or SOX10 immunohistochemistry, and/or those with complete absence of neurofibromin protein expression by immunohistochemistry were diagnosed as malignant nerve sheath tumor upon exclusion of other entities as indicated by clinical setting, tumor location and morphology. All malignant peripheral nerve sheath tumor cases were tested for $\mathrm{t}(\mathrm{X} ; 18)(\mathrm{p} 11.2 ; \mathrm{q11.2})$ via fluorescence in situ hybridization (FISH) and were negative. All synovial sarcoma and fibrosarcomatous dermatofibrosarcoma protuberans cases were diagnosed by one of the study authors (AEH) using the 2013 WHO Classification of Soft Tissue Neoplasms. ${ }^{14,15}$ Molecular confirmation of $t(X ; 18)$ either by FISH or cytogenetics was available for 67 of $82(82 \%)$ synovial sarcomas. All fibrosarcomatous dermatofibrosarcoma protuberans had histologically confirmed synchronous or metachronous cutaneous DFSP. None of the synovial sarcoma or fibrosarcomatous dermatofibrosarcoma protuberans patients had Neurofibromatosis type-1.

\section{Immunohistochemistry}

Immunohistochemical analysis of H3K27me3 was performed using formalin-fixed paraffin-embedded sections on a combination of whole-slide sections and tissue microarrays. Whole-slide sections were used for 10 fibrosarcomatous dermatofibrosarcoma protuberans and 35 of 82 synovial sarcomas. The remaining synovial sarcomas were analyzed in triplicate on a previously constructed tissue microarray using $1 \mathrm{~mm}$ cores. ${ }^{16}$ Malignant peripheral nerve sheath tumors were analyzed in duplicate on tissue microarrays using $2 \mathrm{~mm}$ cores. Four-micron sections were stained using standard techniques on a Leica Bond 3 automated immunostainer (Leica, Buffalo Grove, IL, USA) using a rabbit monoclonal primary antibody (clone C36B11, 1:50 dilution, Cell Signaling Technology, Danvers, MA, USA) and polymeric secondary detection system (Refine, Leica). A malignant peripheral nerve sheath tumor with known H3K27me3 loss was used as a positive control.

Only cases with appropriate internal positive control staining (endothelial cells and inflammatory cells) were scored and included in the study. Nuclear staining for H3K27me3 was scored by two authors (MP and AEH) independently, as follows: 
- 3+: Retained expression in $95 \%$ or more of tumor cells (diffuse staining)

- 2+: Retained expression in 50-95\% of tumor cells (loss in minority)

- 1+: Retained expression in 5-50\% of tumor cells (loss in majority)

- 0: Retained expression in less than $5 \%$ of tumor cells (complete loss)

Any discrepant cases were re-reviewed until a consensus was reached. Furthermore, we also scored tumors simply as either negative or positive (score 0 , or $>0$, respectively, in the 4-tier system). Using the negative-positive, two-tier system, scores were completely concordant after independent review without need for re-review.

\section{Statistical Analysis}

Statistical analyses were performed using STATA 13 Professional package (STATA/MP 14.2, StataCorp, College Station, TX, USA). Significance of associations between malignant peripheral nerve sheath tumor

Table 1 Distribution of age, gender, and tumor location

\begin{tabular}{lccc}
\hline & Male (\%) & $\begin{array}{c}\text { Age median } \\
\text { (range) }\end{array}$ & Extremity (\%) \\
\hline $\begin{array}{l}\text { SS (n=82) } \\
\quad \text { Monophasic } \\
\quad(n=62)\end{array}$ & 55 & $33(8-77)$ & 81 \\
$\quad \begin{array}{l}\text { Biphasic }(n=18) \\
\begin{array}{l}\text { Poorly } \\
\text { differentiated }\end{array}\end{array}$ & 56 & $31.5(9-55)$ & 78 \\
$\quad(n=2)$ & & $35.5(26-45)$ & 50 \\
$\begin{array}{l}\text { MPNST }(n=39) \\
\quad \text { NF1 associated } \\
(n=26)\end{array}$ & 41 & $37(11-72)$ & 41 \\
$\quad$ Sporadic $(n=13)$ & 31 & $35.5(11-60)$ & 35 \\
FS-DFSP $(n=10)$ & 60 & $41(23-72)$ & 46 \\
\hline
\end{tabular}

Abbreviations: FS-DFSP, fibrosarcomatous dermatofibrosarcoma protuberans; MPNST, malignant peripheral nerve sheath tumor; NF1, neurofibromatosis 1; SS, synovial sarcoma. and synovial sarcoma diagnostic groups were compared with Kruskal-Wallis test for continuous data and Fisher's Exact test for categorical data. Given the small sample size for fibrosarcomatous dermatofibrosarcoma protuberans, it is not included to the statistical analyses. Significances of associations between H3K27me3 loss and clinical variables in malignant peripheral nerve sheath tumors were evaluated with univariate logistic regression. Survival statistics were performed using Cox proportional hazards method. Given the lack of significance in the univariate models, multivariate models were not analyzed. A $P$-value of less than 0.05 was accepted as statistically significant.

\section{Results}

The current study consists of 131 primary tumors $(82$ synovial sarcomas, 39 malignant peripheral nerve sheath tumors, and 10 fibrosarcomatous dermatofibrosarcoma protuberans). Distribution of age and gender across tumor types are summarized in Table 1. Malignant peripheral nerve sheath tumor and synovial sarcoma groups were similar with respect to gender distribution and age $(P=0.18$ and $P=0.13$, respectively). Synovial sarcoma was more likely to involve extremities (81\%) while malignant peripheral nerve sheath tumor was more likely to be located in central axial sites $(59 \%, P<0.0001)$. Five malignant peripheral nerve sheath tumors were located in a paraspinal region, two from Neurofibromatosis type-1 patients. Three of the malignant peripheral nerve sheath tumors were graded as 'lowgrade' per morphologic criteria, and all were seen in Neurofibromatosis type- 1 patients.

The distribution of H3K27me3 staining scores are summarized in Table 2. Representative images of H3K27me3 stains for malignant peripheral nerve sheath tumors, synovial sarcoma and dermatofibrosarcoma protuberans are demonstrated in Figures 1, 2 and 3, respectively. The fractional distribution of cells staining was consistent throughout tumors stained as whole-slides (ie little regional variability).

Table 2 Distribution of the H3K27me3 staining scores

\begin{tabular}{|c|c|c|c|c|}
\hline & $0(<5 \%)$ & $1+(5-50 \%)$ & $2+(50-95 \%)$ & $3+(>95 \%)$ \\
\hline$S S(n=82)$ & $7(9 \%)$ & $14(17 \%)$ & $37(45 \%)$ & $24(29 \%)$ \\
\hline Monophasic $(n=62)$ & $7(11 \%)$ & $9(15 \%)$ & $28(45 \%)$ & $18(29 \%)$ \\
\hline Biphasic $(n=18)$ & - & $4(22 \%)$ & $9(50 \%)$ & $5(28 \%)$ \\
\hline Poorly differentiated $(n=2)$ & - & $1(50 \%)$ & - & $1(50 \%)$ \\
\hline MPNST $(n=39)$ & $17(44 \%)$ & $6(15 \%)$ & $4(10 \%)$ & $12(31 \%)$ \\
\hline NF1 associated $(n=26)$ & $13(50 \%)$ & $6(23 \%)$ & - & $7(27 \%)$ \\
\hline Sporadic $(n=13)$ & $4(31 \%)$ & - & $4(31 \%)$ & $5(38 \%)$ \\
\hline FS-DFSP $(n=10)$ & $1(10 \%)$ & $3(30 \%)$ & $3(30 \%)$ & $3(30 \%)$ \\
\hline
\end{tabular}

Abbreviations: FS-DFSP, fibrosarcomatous dermatofibrosarcoma protuberans; H3K27me3, Trimethylation at Lysine 27 of histone 3; MPNST, malignant peripheral nerve sheath tumor; NF1, neurofibromatosis 1; SS, synovial sarcoma. 


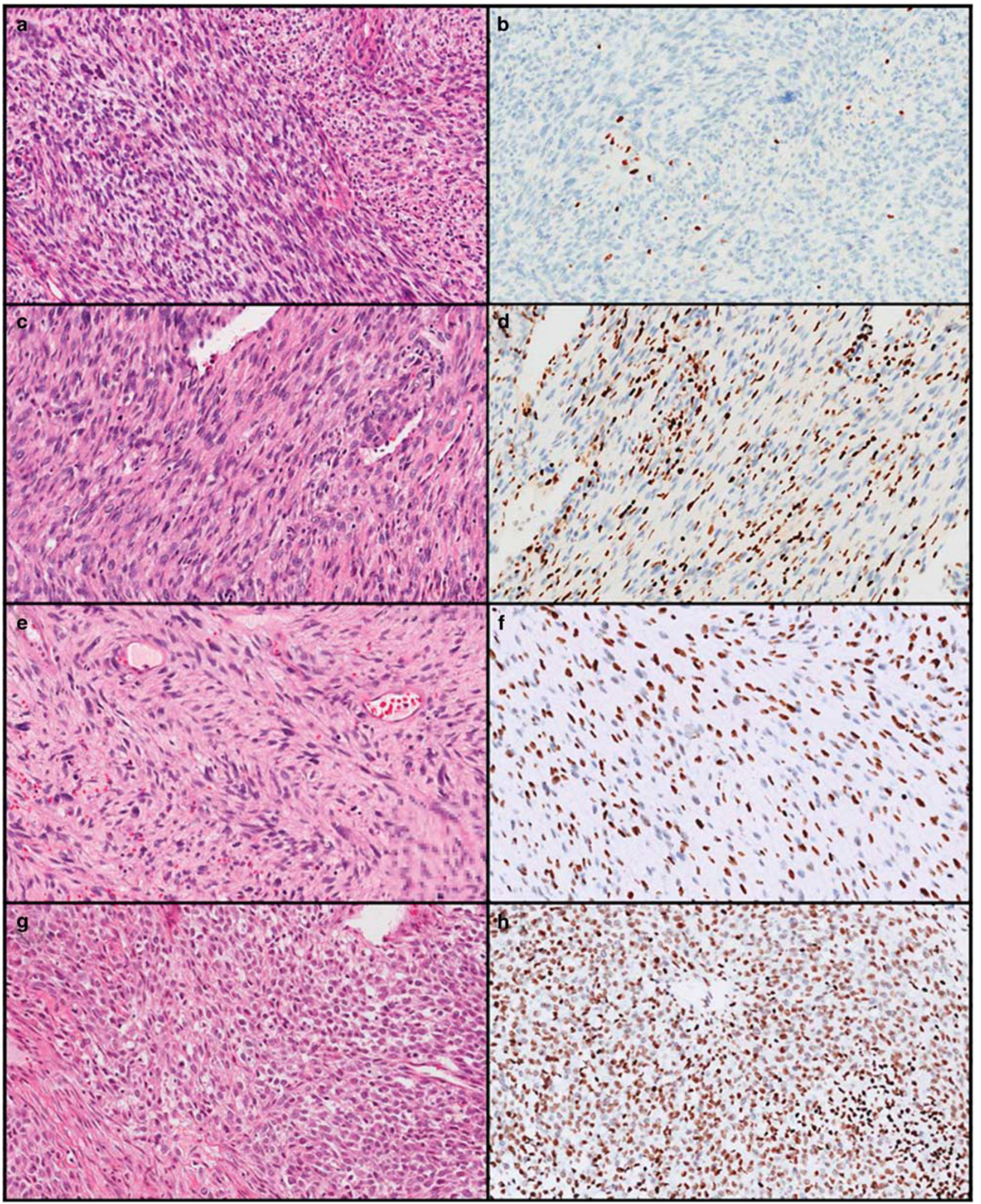

Figure 1 Representative H3K27me3 images among malignant peripheral nerve sheath tumors. (a-d) Hematoxylin-Eosin stain, 20x; (e-h) H3K27me3 stain corresponding to cases in a-d, 20x; (e) retained (Diffuse) H3H27me3 (expression in >95\% of tumor cells), score 3+; (f) expression in $50-95 \%$ of tumor cells, score $2+$; (g) expression in $5-50 \%$ of tumor cells, score $1+$; (h) loss of H3K27me3 (expression in $<5 \%$ of tumor cells), score 0 . 


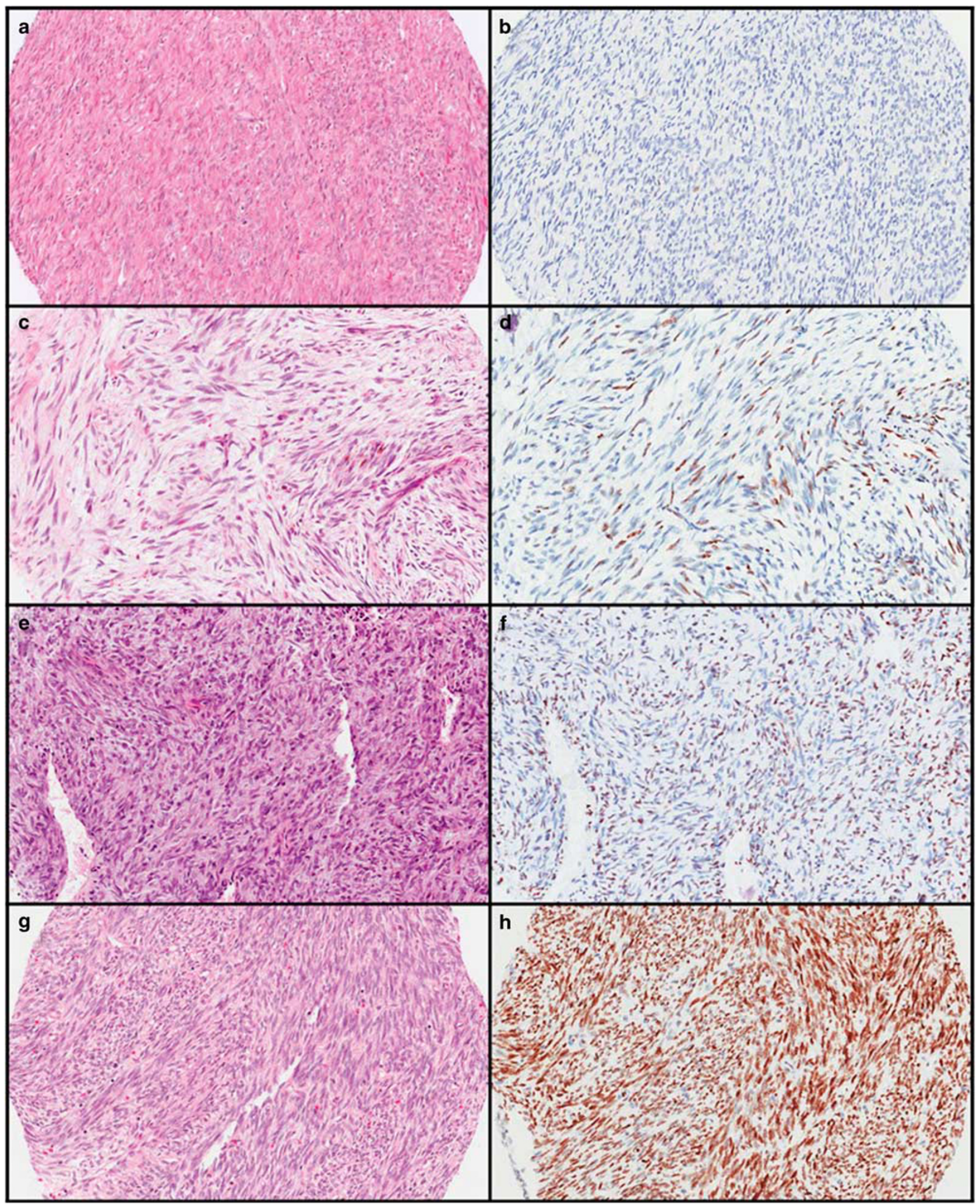

Figure 2 Representative H3K27me3 images among synovial sarcomas. (a-d) Hematoxylin-Eosin stain, 20x; (e-h) H3K27me3 stain corresponding to cases in a-d, 20x; (e) retained (Diffuse) H3H27me3 (expression in $>95 \%$ of tumor cells), score 3+; (f) expression in $50-95 \%$ of tumor cells, score $2+$; (g) expression in $5-50 \%$ of tumor cells, score $1+$; (h) loss of H3K27me3 (expression in $<5 \%$ of tumor cells), score 0. 


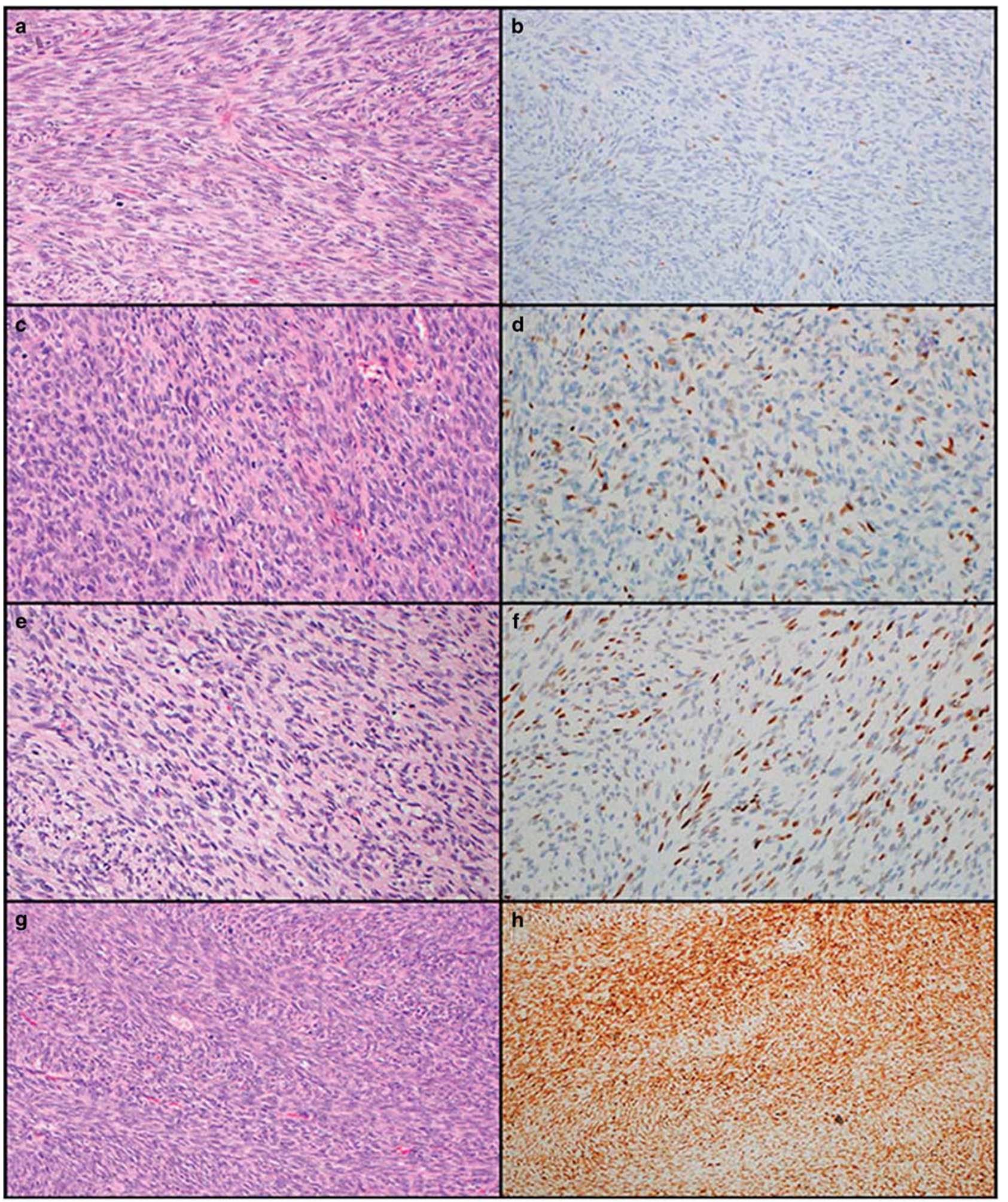

Figure 3 Representative H3K27me3 Images among fibrosarcomatous dermatofibrosarcoma protuberans. (a-d) Hematoxylin-Eosin stain, 20x; (e-h) H3K27me3 stain corresponding to cases in a-d, 20x; (e) retained (Diffuse) H3H27me3 (expression in $>95 \%$ of tumor cells), score 3+; (f) expression in 50-95\% of tumor cells, score 2+; (g) expression in 5-50\% of tumor cells, score 1+; (h) loss of H3K27me3 (Expression in $<5 \%$ of tumor cells), score 0 . 
When data was analyzed using the four-tier scoring system (see Methods), there was a statistically significant difference between synovial sarcoma and malignant peripheral nerve sheath tumor regarding the H3K27me3 staining $(P<0.0001)$. All synovial sarcomas with H3K27me3 loss (score 0) were monophasic; however, this did not reach statistical significance relative to biphasic synovial sarcomas $(P=0.5)$. Using the four-tier scoring system, Neurofibromatosis-associated malignant peripheral nerve sheath tumors were associated with lower staining scores for H3K27me3 as compared to sporadic malignant peripheral nerve sheath tumors $(P=0.007)$. Using the simpler negative-positive scoring system, loss of H3K27me3 expression (score 0 ) was seen in $50 \%$ of sporadic malignant peripheral nerve sheath tumors and 31\% of Neurofibromatosisassociated malignant peripheral nerve sheath tumors $(P=0.25)$. One of 10 fibrosarcomatous dermatofibrosarcoma protuberans $(10 \%)$ showed complete loss (score 0) with three cases each showing 1+,2+, and 3 + staining, respectively (Table 2). Among synovial sarcomas, no significant difference was noted when comparing staining of whole-slide sections to tissue microarrays using either four-tier or negative-positive scoring systems ( $P=0.26$ and 1.00 , respectively).

Using the negative-positive scoring system, the sensitivity and specificity of H3K27me3 loss for the diagnosis of malignant peripheral nerve sheath tumor versus synovial sarcoma was $44 \%$ and $92 \%$, respectively. With these criteria, loss of H3K27me3 staining would have a positive predictive value of $71 \%$ and negative predictive value of $77 \%$ in the differential diagnosis of malignant peripheral nerve sheath tumor versus synovial sarcoma. With these criteria, H3K27me3 loss had a sensitivity of $44 \%$, specificity of $90 \%$, positive predictive value of $94 \%$ and negative predictive value of $29 \%$ in the differential diagnosis of malignant peripheral nerve sheath tumor versus fibrosarcomatous dermatofibrosarcoma protuberans.

Among malignant peripheral nerve sheath tumors, complete loss of H3K27me3 expression (score 0) was not associated with gender, tumor site, or tumor size (Table 3). Of five paraspinal malignant peripheral nerve sheath tumors, two showed complete loss, one showed loss in a minority (50-95\% staining) and two showed diffusely retained staining. Of the three lowgrade malignant peripheral nerve sheath tumors, two showed diffuse staining and one showed complete loss of staining with H3K27me3. Patients with tumors showing H3K27me3 loss were younger than the patients whose tumors retained H3K27me3 expression (CC: $0.016 ; 95 \%$ CI: 0.004-0.028; $P=0.011$ ) and this association remained significant in a multivariate model controlling for Neurofibromatosis status (CC: $0.015 ; 95 \%$ CI: $0.002-0.027 ; P=0.02$ ). There was no statistically significance association between progression-free and overall survival and H3K27me3 status ( $P>0.05$ for both, Figure 4). Survival statistics are summarized in Table 4.
Table 3 Association between clinical parameters and H3K27me3 status in malignant peripheral nerve sheath tumors

\begin{tabular}{|c|c|c|c|c|}
\hline & $\begin{array}{c}\text { Total } \\
\text { (n) }\end{array}$ & $\begin{array}{c}\text { H3К27me3 } \\
\text { loss (Score 0) }\end{array}$ & $\begin{array}{c}\text { H3К27me3 } \\
\text { retained } \\
\text { (Score } 1+\text { or } \\
\text { more) }\end{array}$ & $\mathrm{P}$-value ${ }^{\mathrm{a}}$ \\
\hline \multicolumn{5}{|l|}{ Gender } \\
\hline Male & 16 & 7 (44\%) & $9(56 \%)$ & 0.99 \\
\hline Female & 23 & $10(43 \%)$ & $13(57 \%)$ & \\
\hline \multicolumn{5}{|l|}{ Age (years) } \\
\hline Median \pm s.d. & 39 & $32 \pm 11.6$ & $42.5 \pm 12$ & 0.011 \\
\hline \multicolumn{5}{|l|}{ Tumor type } \\
\hline NF1 associated & 26 & $13(50 \%)$ & $13(50 \%)$ & 0.25 \\
\hline Sporadic & 13 & $4(31 \%)$ & $9(69 \%)$ & \\
\hline \multicolumn{5}{|l|}{ Tumor site } \\
\hline Extremity & 16 & $5(31 \%)$ & $11(69 \%)$ & 0.19 \\
\hline Non-extremity & 23 & $12(52 \%)$ & $11(48 \%)$ & \\
\hline Paraspinal & 5 & $2(40 \%)$ & $3(60 \%)$ & 0.86 \\
\hline Non-paraspinal & 34 & $15(44 \%)$ & $19(56 \%)$ & \\
\hline \multicolumn{5}{|l|}{ Tumor size $(\mathrm{cm})$} \\
\hline Median \pm SD & 37 & $9.5 \pm 5.9$ & $7.5 \pm 7$ & 0.28 \\
\hline
\end{tabular}

Abbreviation: H3K27me3, trimethylation at Lysine 27 of histone 3.

${ }^{a} P$-values are based on univariate logistic regression test.
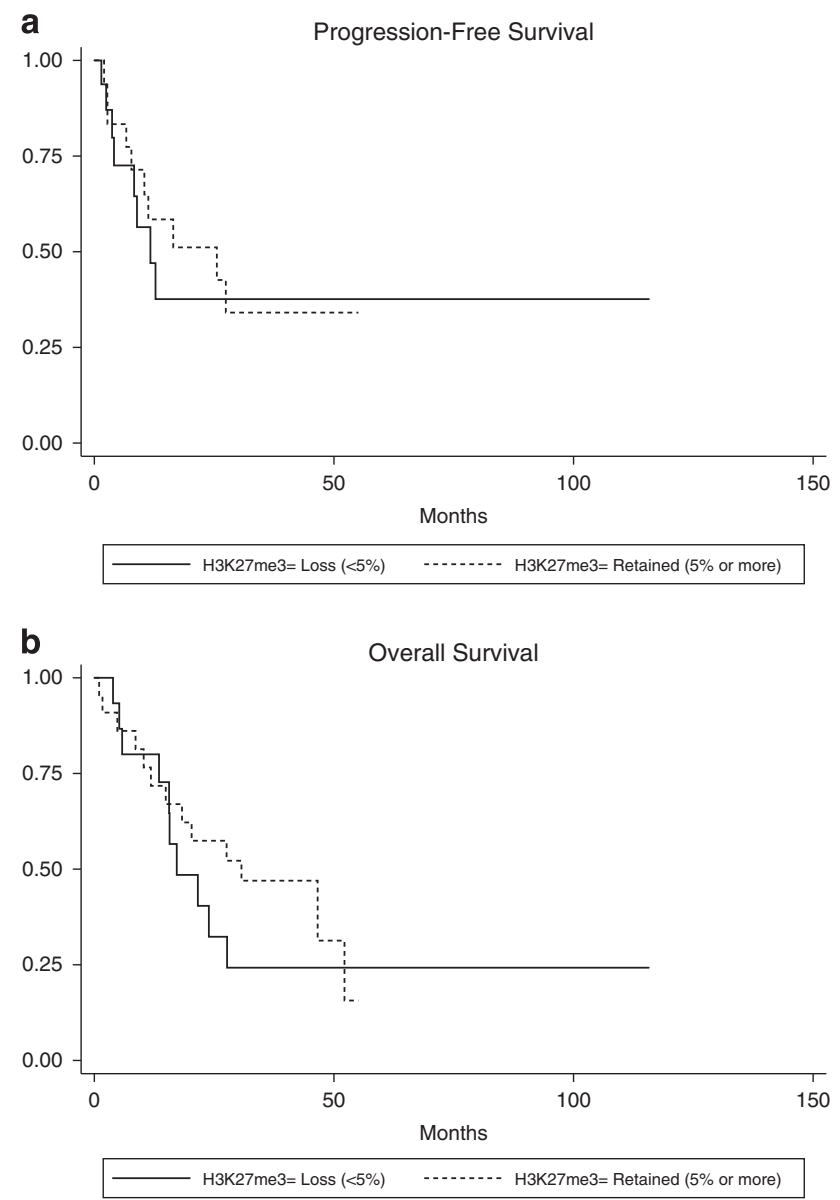

Figure 4 Kaplan-Meier survival curves for progression-free survival and overall survival among malignant peripheral nerve sheath tumors based on H3K27me3 status. (a) Progression-free survival; (b) overall survival. 
Table 4 Association of H3K27me3 status with progression-free and overall survival among malignant peripheral nerve sheath tumors

\begin{tabular}{lcc}
\hline & $\begin{array}{c}\text { H3K27me3 retained } \\
(\mathrm{n}=22)\end{array}$ & $\begin{array}{c}\text { H3K27me3 loss } \\
(\mathrm{n}=17)\end{array}$ \\
\hline Percent recurred & $45.5 \%$ & $47.1 \%$ \\
$\begin{array}{l}\text { Median PFS } \\
\text { (95\% CI), months }\end{array}$ & $25.6 \pm 8.8(7.7-\mathrm{NA})$ & $11.7 \pm 2.8(3.7-\mathrm{NA})$ \\
$\begin{array}{l}\text { HR (95\% CI) for PFS } \\
P \text { (for PFS) }\end{array}$ & Reference & $1.23(0.48-3.14)$ \\
Percent deceased & $59.1 \%$ & 0.668 \\
$\begin{array}{l}\text { Median OS } \\
\text { (95\% CI), months }\end{array}$ & $30.7 \pm 9.3(11.8-52.2)$ & $17.2 \pm 5.1(5.8-27.7)$ \\
$\begin{array}{l}\text { HR (95\% CI) for OS } \\
P \text { (for OS) }\end{array}$ & Reference & $1.30(0.56-2.98)$ \\
& & 0.540 \\
\hline
\end{tabular}

Abbreviations: CI, confidence interval; HR, hazard ratio; H3K27me3, Trimethylation at Lysine 27 of histone 3; OS, overall survival; PFS, progression-free survival.

\section{Discussion}

The diagnosis of malignant peripheral nerve sheath tumor can be challenging, especially outside the Neurofibromatosis setting due to relatively nonspecific histologic features and imperfect sensitivity and specificity of currently available ancillary immunohistochemistry. ${ }^{3,4}$ Loss of H3K27me3 expression has been reported as a useful diagnostic marker of malignant peripheral nerve sheath tumor. However, the sensitivity and specificity values, particularly with respect to synovial sarcoma and fibrosarcomatous dermatofibrosarcoma protuberans, vary among prior studies. In the present study, we have investigated the utility of H3K27me3 staining using a monoclonal antibody in the diagnosis of malignant peripheral nerve sheath tumor relative to synovial sarcoma and fibrosarcomatous dermatofibrosarcoma protuberans.

Here, we have demonstrated that partial loss of H3K27me3 staining is common in synovial sarcoma and fibrosarcomatous dermatofibrosarcoma protuberans, as well as in malignant peripheral nerve sheath tumor. This is in agreement with some prior studies. Partial loss of H3K27me3 staining (or mosaic loss as it was referred in some studies) has been reported in $17 \%$ of malignant peripheral nerve sheath tumors and $38 \%$ of other spindle cell neoplasms in the differential diagnosis of malignant peripheral nerve sheath tumor. ${ }^{11}$ Partial loss may be biologically significant since a previous study showed that four of four Neurofibromatosisassociated malignant peripheral nerve sheath tumors with mosaic staining carry heterozygous mutations either in SUZ12 or EED. ${ }^{8}$ Nevertheless, partial loss is more difficult to score accurately than complete absence or presence of staining especially when dealing with an isolated case rather than comparing a large series of tumors for a research study. For these reasons, we propose that partial H3K27me3 not be utilized to support the diagnosis of malignant peripheral nerve sheath tumor over morphologic mimics.

On the other hand, we have confirmed that $44 \%$ of malignant peripheral nerve sheath tumors show complete loss (score 0) of H3K27me3 immunoreactivity, compared to $9 \%$ for synovial sarcomas and $10 \%$ for fibrosarcomatous dermatofibrosarcoma protuberans. Thus, complete loss may be diagnostically useful to support malignant peripheral nerve sheath tumor in this differential diagnosis. The complete agreement of the authors' independent staining score using a negative-positive system also suggests that such a simpler scoring system would be more practical and less hindered by interobserver variability in routine use. Arguably, the relatively low sensitivity means that retained or mosaic H3K27me3 staining should not deter one from rendering a diagnosis of malignant peripheral nerve sheath tumor. The frequency of loss of H3K27me3 expression is lower than the previously reported range in the literature. ${ }^{7-11}$ Some of these studies reported results based on polyclonal antibodies used in a wide range of dilutions (1:500-1:8000), which may have influenced the results, especially for the evaluation of cases with mosaic staining. Other studies used the same monoclonal antibody as the current study, albeit at slightly lower antibody concentrations than ours (1:200 versus 1:50).

We have found that loss of H3K27me3 is more frequent among sporadic malignant peripheral nerve sheath tumors (50\%) as compared to Neurofibromatosis-associated malignant peripheral nerve sheath tumors (31\%) but this difference was not statistically significant $(P=0.25)$. This is similar to previous studies using a monoclonal antibody for their immunohistochemical analysis. ${ }^{8,11}$ It is also in concordance with some molecular studies demonstrating more frequent loss of function mutations in PRC2 components (EED or SUZ12) among sporadic as compared to Neurofibromatosis-associated malignant peripheral nerve sheath tumors. ${ }^{6}$ On the other hand, others reported higher rate of loss of staining among Neurofibromatosis-associated cases using a polyclonal antibody. ${ }^{7,9,10}$ One study comparing the results of monoclonal antibody with the polyclonal antibody reported discordant results in only two of 52 tumors, with the monoclonal antibody associated with crisper staining and less background. ${ }^{8}$ Another group reported that the polyclonal antibody showed at least mosaic staining in 9 of 30 cases that were negative with the monoclonal antibody; however, they did not specify whether discordant cases were Neurofibromatosis-associated cases. ${ }^{11}$ Taken together, the above results suggest, albeit not conclusively, that differences in antibody choice and dilution may explain some of the differences in reported H3K27me3 in 
sporadic and Neurofibromatosis-associated malignant peripheral nerve sheath tumors.

The rate of H3K27me3 loss in synovial sarcoma varies in the literature. Some studies, using both monoclonal and polyclonal antibodies, reported that no synovial sarcoma showed complete H3K27me3 loss. ${ }^{8,9,11}$ However, others reported complete loss of H3K27me3 staining in up to $60 \%$ of synovial sarcomas. ${ }^{10}$ The latter group considered any staining weaker than the internal positive control as loss and used relatively low antibody concentration, which may contribute to their higher reported rate of loss. ${ }^{10}$ Paradoxically, however, the rate of loss in malignant peripheral nerve sheath tumors (34\%) in the above study is among the lowest in the literature (ie H3K27me3 was retained in more malignant peripheral nerve sheath tumors than other studies). Thus, low antibody sensitivity or detection signal may not entirely explain the higher frequency of H3K27me3 loss in synovial sarcoma. In fact, it was this discrepancy that prompted the present study. Based on our results, from a practical perspective, we propose that complete loss of H3K27me3 is 'relatively specific' for the diagnosis of malignant peripheral nerve sheath tumor compared with synovial sarcoma. As with any ancillary test however, H3K27me3 must be interpreted with caution and interpreted only within the context of other ancillary tests.

While the loss of H3K27me3 expression in malignant peripheral nerve sheath tumor has a clear genetic basis, due to SUZ12 and EED mutations, the mechanism for H3K27me3 loss in synovial sarcoma and fibrosarcomatous dermatofibrosarcoma protuberans is less clear. The PRC2 complex, composed of a trimeric core of SUZ12, EED and EZH1/2, is normally responsible for di- and trimethylation of H3K27. Activating mutations and amplifications of EZH2 have been reported in various lymphomas, melanoma, lung and prostate carcinoma, and loss-of-function alterations are seen in various leukemias and myelodysplastic syndrome. ${ }^{17}$ To the best of our knowledge, there are no reports of EZH mutations in synovial sarcoma, however; synovial sarcoma has been associated with alterations in EZH2 expression through epigenetic mechanisms. ${ }^{18,19}$ The characteristic alteration of synovial sarcoma, SYT-SSX oncogene due to t(X;18)(p11.2; q11.2) has been associated with both aberrant silencing and activation of target genes through interaction with SWI/SNF and polycomb complexes. One study showed that SYT-SSX2 occupies H3K27me3-labeled regions within or near $72 \%$ of positively regulated and $44 \%$ of negatively regulated genes. ${ }^{18}$ While they reported that the location and extent of H3K27me3 were variable across all genes, they did not comment about the global expression levels of H3K27me3, which would correlate with immunohistochemistry results. It is possible that global H3K27me3 levels are reduced, despite increased H3K27me3 signature in some genetic loci. A similar mechanism was recently reported in a subset of posterior fossa ependymomas. ${ }^{20}$
Additional studies assessing the methylation profiles in synovial sarcomas are necessary to further explore this possibility or identify the molecular basis of decreased H3K27me3 expression in a subset of cases.

Asano et al. suggested that malignant peripheral nerve sheath tumors with H3K27me3 loss were associated with worse progression-free survival, although there was no association between H3K27me3 status and overall survival. ${ }^{11}$ While they accepted a $P$-value of 0.068 as significant, this would be interpreted by others as merely a trend towards worse progression-free survival. In our dataset, we were not able to show any association between the loss of H3K27me3 staining and progression-free or overall survival among malignant peripheral nerve sheath tumors, although our cohort was relatively small.

In summary, complete loss of H3K27me3 immunostaining can be a useful tool in the diagnosis of malignant peripheral nerve sheath tumor in this differential diagnosis setting since it offers moderate sensitivity and relatively high specificity. It must be remembered that retention of H3K27me3 does not exclude the diagnosis of malignant peripheral nerve sheath tumor. Partial loss of H3K27me3 is not uncommon in other spindle cell neoplasms in the differential diagnosis of malignant peripheral nerve sheath tumor, and its diagnostic utility for malignant peripheral nerve sheath tumor is therefore limited.

\section{Acknowledgments}

This study was supported by the Residents' Teaching and Research Endowment from the University of California San Francisco, Department of Anatomic Pathology.

\section{Disclosure/conflict of interest}

The authors declare no conflict of interest.

\section{References}

1 Toro JR, Travis LB, Wu HJ, et al. Incidence patterns of soft tissue sarcomas, regardless of primary site, in the surveillance, epidemiology and end results program, 1978-2001: an analysis of 26,758 cases. Int J Cancer 2006;119:2922-2930.

2 Rodriguez FJ, Folpe AL, Giannini C, et al. Pathology of peripheral nerve sheath tumors: diagnostic overview and update on selected diagnostic problems. Acta Neuropathol 2012;123:295-319.

3 Kang Y, Pekmezci M, Folpe AL, et al. Diagnostic utility of SOX10 to distinguish malignant peripheral nerve sheath tumor from synovial sarcoma, including intraneural synovial sarcoma. Mod Pathol 2014;27:55-61.

4 Karamchandani JR, Nielsen TO, van de Rijn M, et al. Sox10 and S100 in the diagnosis of soft-tissue neoplasms. Appl Immunohistochem Mol Morphol 2012;20:445-450. 
5 Zhang Q, Padi SK, Tindall DJ, et al. Polycomb protein EZH2 suppresses apoptosis by silencing the proapoptotic miR-31. Cell Death Dis 2014;5:e1486.

6 Lee W, Teckie S, Wiesner T, et al. PRC2 is recurrently inactivated through EED or SUZ12 loss in malignant peripheral nerve sheath tumors. Nat Genet 2014;46: 1227-1232.

7 Rohrich M, Koelsche C, Schrimpf D, et al. Methylationbased classification of benign and malignant peripheral nerve sheath tumors. Acta Neuropathol 2016;131: 877-887.

8 Prieto-Granada CN, Wiesner T, Messina JL, et al. Loss of H3K27me3 expression is a highly sensitive marker for sporadic and radiation-induced MPNST. Am J Surg Pathol 2016;40:479-489.

9 Schaefer IM, Fletcher CD, Hornick JL. Loss of H3K27 trimethylation distinguishes malignant peripheral nerve sheath tumors from histologic mimics. Mod Pathol 2016;29:4-13.

10 Cleven AH, Sannaa GA, Briaire-de Bruijn I, et al. Loss of H3K27 tri-methylation is a diagnostic marker for malignant peripheral nerve sheath tumors and an indicator for an inferior survival. Mod Pathol 2016;29: $582-590$.

11 Asano N, Yoshida A, Ichikawa H, et al. Immunohistochemistry for trimethylated H3K27 in the diagnosis of malignant peripheral nerve sheath tumours. Histopathology 2017;70:385-393.

12 Pekmezci M, Reuss DE, Hirbe AC, et al. Morphologic and immunohistochemical features of malignant peripheral nerve sheath tumors and cellular schwannomas. Mod Pathol 2015;28:187-200.

13 Reuss DE, Perry A, Louis DN, et al. Malignant Peripheral Nerve Sheath Tumour. In: Louis DN, Ohgaki H, Wiestler OD, Cavenee WK (eds). WHO Classification of Tumours of the Central Nervous System. Revised 4th edn. International Agency for Research on Cancer: Lyon, 2016, pp 226-229.

14 Fisher C, de Brujin DRH, Geurts van Kessel A. Synovial Sarcoma. In: Fletcher CDM, Bridge JA, Hogendoorn P, Mertens F (eds). Who Classification of Tumours of Soft Tissue and Bone 4th edn. International Agency for Research on Cancer: Lyon, 2013, pp 200-204.

15 Mentzel T, Pedeutour F, Lazar A, et al. Dermatofibrosarcoma protuberans. In: Fletcher CDM, Bridge JA, Hogendoorn P, Mertens F (eds). WHO Classification of Tumours of Soft Tissue and Bone 4th edn. International Agency for Research on Cancer: Lyon, 2013, pp 77-79.

16 Horvai AE, Kramer MJ, O'Donnell R. Beta-catenin nuclear expression correlates with cyclin D1 expression in primary and metastatic synovial sarcoma: a tissue microarray study. Arch Pathol Lab Med 2006;130:792-798.

17 Souroullas GP, Jeck WR, Parker JS, et al. An oncogenic Ezh2 mutation induces tumors through global redistribution of histone 3 lysine 27 trimethylation. Nat Med 2016;22:632-640.

18 Garcia CB, Shaffer CM, Eid JE. Genome-wide recruitment to Polycomb-modified chromatin and activity regulation of the synovial sarcoma oncogene SYTSSX2. BMC Genomics 2012;13:189.

19 Changchien YC, Tatrai P, Papp G, et al. Poorly differentiated synovial sarcoma is associated with high expression of enhancer of zeste homologue 2 (EZH2). J Transl Med 2012;10:216.

20 Bayliss J, Mukherjee P, Lu C, et al. Lowered H3K27me3 and DNA hypomethylation define poorly prognostic pediatric posterior fossa ependymomas. Sci Transl Med 2016;8:366ra161. 